\title{
Debate
}

\section{Is primary care ready to take on Attention Deficit Hyperactivity Disorder?}

\author{
Ajay Thapar*1 and Anita Thapar ${ }^{2}$
}

Address: ${ }^{1}$ Department of General Practice, University of Wales College of Medicine and General Practitioner, Taff Riverside Practice, Cardiff, Wales and ${ }^{2}$ Child and Adolescent Psychiatry Section, Department of Psychological Medicine, University of Wales College of Medicine, Cardiff, Wales

E-mail: Ajay Thapar* - ajay.thapar@net.ntl.com; Anita Thapar - thapar@cardiff.ac.uk

${ }^{*}$ Corresponding author

Published: 16 April 2002

BMC Family Practice 2002, 3:7
Received: II February 2002

Accepted: 16 April 2002

This article is available from: http://www.biomedcentral.com/I47I-2296/3/7

(C) 2002 Thapar and Thapar; licensee BioMed Central Ltd. Verbatim copying and redistribution of this article are permitted in any medium for any purpose, provided this notice is preserved along with the article's original URL.

\begin{abstract}
Background: Attention Deficit Hyperactivity Disorder (ADHD) is a common childhood psychiatric disorder. The management of ADHD has recently been highlighted. The National Institute of Clinical Excellence (NICE) and Scottish Intercollegiate Guidelines network (SIGN) have both produced management guidelines. Doctors working within Primary Care in countries such as the United States play an important role in the management of ADHD. In the United Kingdom however the role of doctors in primary care in the management of ADHD, both individually and within shared care protocols, is only now being identified and defined. Is this role for Primary Care likely to be acceptable and effective?
\end{abstract}

Discussion: There is some evidence that doctors working within Primary Care in the United Kingdom are willing to follow up children on medication for ADHD and carry out monitoring of physical status. However many feel unconfident in the management of ADHD and most have received little or no training in child psychiatry. There are also concerns that adverse media reports will have an undue influence on the attitudes of doctors within primary care to families with children suffering from ADHD.

Summary: There are important barriers to be tackled before shared care protocols for ADHD can be successfully implemented in the United Kingdom. Tailored information about ADHD needs to be provided to doctors in primary care. Clear dialogue between planners and healthcare professionals from both primary and secondary care is essential to ensure that service delivery is acceptable to healthcare providers, tailored to their skills and is adequately resourced.

\section{Background}

Attention Deficit Hyperactivity Disorder (ADHD), known as Hyperkinetic Disorder under the ICD-10 classificatory system, is a common disorder that affects between 1.5\% and $6 \%$ of children $[1,2]$. The disorder is an early onset condition that is associated with educational failure, social difficulties and an increased risk of antisocial behav- iour and problems in adult life including substance misuse and criminality [1]. ADHD now represents the commonest reason for follow up in child and adolescent psychiatry clinics [2] and an average general practitioner can expect to have between two and four children receiving treatment for it on their list. Nevertheless there is evi- 
dence that most children who fulfil diagnostic criteria remain undetected in the general population [3].

The treatment of ADHD has been the focus of extensive review and several authoritative reports and recommendations have been recently circulated [4-8]. These recommendations support the use of stimulant medication (such as methylphenidate (Ritalin, Equasym) and dexamphetamine (dexedrine)) as first line treatment (either alone or in combination with non-pharmacological interventions including behaviour therapy) for children with accurately diagnosed ADHD [4-9]. Moreover the high level of under treatment has also been highlighted in the recent National Institute of Clinical Excellence (NICE) guidance on the use of methylphenidate in ADHD [4].

\section{Discussion}

Current recommendations are that medication should be initiated by child and adolescent psychiatrists or paediatricians or learning disability specialists with particular expertise $[4,5]$. Once the condition is stabilised children can then be followed up in primary care $[4,5]$ and NICE guidance recommends health authorities should draw up shared care protocols [4]. Is primary care in this country ready to follow these recommendations? Follow up of ADHD is often undertaken by primary care in the Unites States $[8,10]$. Moreover adult ADHD is becoming increasingly recognised and some of the cohort of children and adolescents who are currently on stimulant medication will graduate to adult mental health services. These factors together with statements in the NICE guidance on the use of methylphenidate are likely to increase pressure on primary care to become more involved in the management of ADHD.

However there is a great need for caution and careful planning before implementing shared care protocols for ADHD and sharing follow up with primary care. Primary care teams are already having to assume responsibility for follow up of an increasing number of chronic conditions and although for some (such as diabetes) there is evidence this is successful [11], the monitoring of children on treatment for ADHD may pose different problems. Most general practitioners and nurses will have undergone no or very little training in child and adolescent psychiatry. Many general practitioners do not feel confident about their skills in recognising and undertaking follow-up management of ADHD and feel that education is essential [12]. Attitudes amongst members of the primary care team members may also represent a problem. The media represents an important source of knowledge of ADHD for many general practitioners [12]. Given that recent media features have highlighted problems with over diagnosis and over treatment this is likely to affect attitudes within the primary care team to the use of stimulant med- ication and heighten anxieties about management. Although there is some evidence that most general practitioners are willing to take on physical monitoring of children on medication for ADHD (e.g. height, weight and blood tests if necessary) the only available data suggest general practitioners feel clinical monitoring of these children is a specialist responsibility [12]. Therefore shared care protocols for ADHD may have limited impact in reducing specialist workload and will need to incorporate effective mechanisms for close integration and good communication between services to ensure that patients and their families receive appropriate care.

It is therefore important that a clear strategy for the follow up care of children with ADHD is devised. Planners need to ensure that roles and responsibilities of different healthcare sectors in monitoring children with ADHD on medication are clearly demarcated and acceptable to the professionals concerned. When these clear roles have been identified, it is then important to ensure that professionals possess the appropriate skills and knowledge to effectively undertake the assigned role. The resource implications of carrying out this monitoring also need to be addressed so that this can be done successfully (as has been achieved for monitoring of anticoagulant therapy). It is essential that patient needs are met in a coordinated and supported manner to avoid a situation of placing children and families into a "no mans land" of uncertain responsibility and inadequate skills,

\section{Summary}

ADHD is likely to become increasingly important for primary care. To ensure that patient care is not compromised by the introduction of shared care protocols it is important that there is a clear dialogue between planners and healthcare professionals from both primary and secondary care. This is necessary to ensure mechanisms are in place for this care to be undertaken effectively and properly organised. Service delivery needs to be sensitive to patient need, acceptable to healthcare providers and tailored to their skills and finally must be adequately resourced.

\section{Authors' contributions}

Both authors (AKT and AT) jointly initiated this paper, researched the topic and contributed to drafting and final preparation of this article.

\section{Declaration of competing interests}

AT (Anita Thapar) has organised academic meetings that have received sponsorship from Celltech (manufacturers of Equasym) and Janssen Cilag (manufacturers of Concerta). 


\section{Acknowledgements}

We wish to thank Professor Eric Taylor for his helpful comments on this paper and Professors' Richard Harrington and Martin Roland for reviewing the manuscript.

\section{References}

I. Taylor E: Syndromes of Attention deficit and overactivity. In Child and Adolescent Psychiatry: modern approaches (Edited by: M Rutter, E Taylor, L Hersov). London: Blackwell Scientific Publications 1994, 285307

2. Meltzer H, Gatward R, Goodman R, Ford T: "Mental health of children and adolescents in Great Britain" London, The Stationery Office, 2000

3. Taylor E, Hemsley R: Treating hyperkinetic disorders in childhood. BMJ 1995, 3 1 0:1617-8

4. National Institute for Clinical Excellence: Guidance on the use of methylphenidate (Ritalin, Equasym) for Attention Deficit/ Hyperactivity Disorder (ADHD) in childhood. Technology Appraisal Guidance No. 13 [http://nice.org.uk]

5. Attention Deficit and Hyperkinetic Disorders in Children and Young People. Sign Publication number 52 (200I) Scottish Intercollegiate Guidelines Network. [http://www.sign.ac.uk]

6. Hill P, Taylor E: An auditable protocol for Attention Deficit/ Hyperactivity Disorder. Archives of Diseases in Childhood 2001, 84:404-409

7. Practice parameters for the use of stimulant medications in the treatment of children, adolescents and adults. Journal of the American Academy of Child and Adolescent Psychiatry supplement. 2002, 41:26S-49S

8. American Academy of Paediatrics Clinical Practice Guideline: Treatment of the School-Aged Child With Attention-Deficit/Hyperactivity Disorder. Paediatrics 200 I, 108(4): 1033-38

9. Taylor E: Development of Clinical Services for Attention-Deficit/ Hyperactivity disorder. Arch. Gen Psychiatry 1999, 56:10971110

10. Levy F: Attention deficit hyperactivity disorder. BM] 1997 , 3 1 5:894-5

II. Diabetes Integrated Care Evaluation Team Integrated care for diabetes: clinical, psychosocial, and economic evaluation. BMJ 1994, 308:1208-12

12. Ball C: Attention deficit hyperactivity disorder (ADHD) and the use of methylphenidate: a survey of the views of General Practitioners Psychiatric Bulletin 200I, 25:30I-304

\section{Pre-publication history}

The pre-publication history for this paper can be accessed here:

http://www.biomedcentral.com/1471-2296/3/7/prepub
Publish with BioMed Central and every scientist can read your work free of charge

"BioMedcentral will be the most significant development for disseminating the results of biomedical research in our lifetime." Paul Nurse, Director-General, Imperial Cancer Research Fund

Publish with BMC and your research papers will be:

- available free of charge to the entire biomedical community

- peer reviewed and published immediately upon acceptance

- cited in PubMed and archived on PubMed Central

- yours - you keep the copyright 